\title{
Assessment of Fall Risk in Elderly Rural Population
}

Jeganathan GEETHA ${ }^{\mathrm{a}}$, Varatharajan SAKTHIVADIVEL ${ }^{\mathrm{b}}$, Archana GAUR

aDepartment of General Medicine, Karpaga Vinayaga Institute of Medical Sciences and Research Center, Maduranthgam, Tamilnadu, India

bepartment of General Medicine, All India Institute of Medical Sciences, Bibinagar, Hyderabad, Telangana, India

'Department of Physiology, All India Institute of Medical Sciences, Bibinagar, Hyderabad, Telangana, India

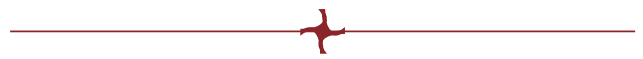

\begin{abstract}
-ABSTRACT
Background: Fall risk assessment is an essential component of a comprehensive fall reduction and prevention program. Fall risk assessment is done to identify patients' risk for falls and ultimately prevent falls from occurring. The aim of the study was to identify the risk factors for fall in the elderly population.

Methods: This study included 250 elderly patients $>60$ years (105 males, 145 females) attending Medicine OPD in a tertiary care hospital. Detailed history including the previous h/o falls, medication history elicited and divided study subjects into two groups: fallers and non-fallers. Cardiovascular, neurological, and musculoskeletal system examinations were done. Handgrip, 30 seconds chair standing test (CST), "timed up and go" test (TUG), and performance-oriented mobility assessment (POMA) were performed.

Results: The prevalence of falls in our study was $27.6 \%$. The mean age of fallers was $72.22 \pm 6.219$ years $(P$ 0.002). Females had significant falls compared to males $(p<0.001)$. In binary logistic regression, age $>80$ [OR-3.8, CI (1.8-8.1); $p$ 0.016], female gender [OR-19.2, CI (3.03-122.2); $p$ 0.002], visual impairment [OR-8.9, CI (1.4-55.4); $p$ 0.019], postural hypotension [OR-59.8, CI (3.3-1.07); $p$ 0.006] and presence of more than three risk factors [OR-9.6, CI (4.6-2.01); $p$ 0.000] were significantly associated with fall in our study.

Conclusion: Falls among older people were common, especially among individuals aged over 80. Females were more prone to fall. The number of risk factors, visual impairment, and postural hypotension increased the fall risk. Simple clinical assessment can identify high-risk patients.
\end{abstract}

Keywords: fall, elderly, risk factor, TUG, POMA.

Address for correspondence:

Dr. Varatharajan Sakthivadivel, MD, Associate Professor

Department of General Medicine, AIIMS- Bibinagar, Hyderabad, Telangana, India, Pin-508126

Tel.: 91-9962528811; email: vsakthivadivel28@gmail.com

Article received on the $26^{\text {th }}$ of September 2021 and accepted for publication on the $10^{\text {th }}$ of December 2021 


\section{INTRODUCTION}

1 ging is a normal physiological process. Aging above 60 years is defined as elderly by Indian criteria (1). An older population will reach two billion people by 2050, out of which $80 \%$ will be in developing countries (2). In India, geriatric population varies from $6.7 \%$ to $12.6 \%$ and will reach 173.1 million by 2026 (3).

Immobility, instability (falls), incontinence, intellectual impairment, and iatrogenic problems are the five common problems seen in the elderly. Among these, falls are a major problem (4). A fall is an event that results in a person coming to rest inadvertently on the ground or floor or other lower level. "Repeated falls" was defined as more than two falls (5). Fall rate increases in proportion to aging leading to excess morbidity and mortality. Falls are the second common cause of mortality worldwide, out of which $60 \%$ is seen in Southeast Asia. In 2005, falls contributed $3.2 \%$ of unintentional mortality in India (6). Prevalence of falls in India ranges from $14 \%$ to $53 \%(7-10)$.

Falls in the elderly population depend on various epidemiological and socio-demographic factors. Fall risk has been related to a several factors such as the history of falls, muscle weakness, gait disturbance, balance deficit, use of an assistive device, visual impairment, mobility impairment, fear of falling, cognitive impairment, depression, sedentary behaviour, age, number of medications, psychotropic, cardiovascular medications, nutritional deficits, urinary incontinence, arthritis, home hazards and footwear (11). Falls are associated with increased morbidity and mortality in the elderly population. They cause poorer overall functioning and lead to early admission to long-term care facilities (12). Fall risk reduction in the elderly is highly essential to avoid these adverse events. So, the present study was conceived to assess the risk factors for fall in the elderly rural population.

\section{MATERIALS AND METHODS}

\section{tudy population and design}

The present hospital-based cross sectional study was conducted at the General Medicine department of a tertiary care center after obtaining ethical approval (KIMS/F/2019/23). A to- tal of 250 elderly patients (105 males and 145 females) was enrolled after obtaining written informed consent. Patients with acute illness, acute painful lower limb conditions, dementia, severe depression, and those who are not willing were excluded from the study.

Detailed history regarding the history of falls, arthritis, fractures, visual, hearing impairment, urinary incontinence and other co-morbidities (diabetes mellitus, hypertension, coronary artery disease, bronchial asthma, chronic obstructive airway disease, chronic kidney disease, and acid peptic disease) were asked. History of polypharmacy was noted; which is defined as taking more than five medications in a month (13). Body mass index (BMI) was measured as per WHO criteria for Asian population (14). Vitals were measured. The cardiovascular, neurological, and musculoskeletal examination was done.

Gait and balance were assessed by the "timed up and go" test (TUG) and performance-oriented mobility assessment (POMA) (15). 30 seconds chair standing test and handgrip were assessed as per protocol $(16,17)$.

The study population was divided into two groups as faller and non-faller depending on the history of fall. Those who had one or more falls were included in the faller group.

\section{Statistical analysis}

The data was analysed using SPSS 22.0. Quantitative variables were represented as mean \pm SD. Categorical data were presented as numbers and percentages. The Chi-square test was used to find the difference among the categorical data. Binary logistic regression analysis was done to analyse the association of risk factors between faller and non-faller groups. The P-value of less than 0.05 was considered statistically significant. $\square$

\section{RESULTS}

ut of 250 patients, 105 were males and 145 females. Subjects' mean age was $67.5 \pm 6$ years. The mean age of the faller group was $72.22 \pm 6.219$ years, significantly higher than that of the non-faller group. Age $>80$ was found to be a significant risk factor for fall (15.9\%). Women had more falls $(75.4 \%)$ than men. The presence of fractures $(30.4 \%)$, visual impairment $(55.1 \%)$, arthritis $(69.6 \%)$, depression $(24.6 \%)$, incontinence $(32.9 \%)$, and postural hypotension 
TABLE 1. Characteristics of study population

\begin{tabular}{|l|l|l|l|}
\hline Parameter & $\begin{array}{l}\text { Fallers }(\geq \mathbf{1} \text { fall }) \\
\text { N=69 }\end{array}$ & $\begin{array}{l}\text { Non-fallers } \\
\text { N=181 }\end{array}$ & p value \\
\hline Age & $72.22 \pm 6.219$ & $70.36 \pm 4.668^{*}$ & 0.011 \\
\hline Sex & & & 0.001 \\
\hline Male & $17(24.6 \%)$ & $88(48.6 \%)^{* *}$ & \\
\hline Female & $52(75.4 \%)$ & $93(51.4 \%)$ & \\
\hline BMI & $21.64 \pm 2.128$ & $22.23 \pm 2.290$ & 0.063 \\
\hline$\geq$ three comorbidities & $1(15.9 \%)$ & $12(6.6 \%)^{*}$ & 0.029 \\
\hline Age $>80$ & $11(15.9 \%)$ & $2(1.1 \%)^{* *}$ & 0.000 \\
\hline Fractures & $21(30.4 \%)$ & $0 * *$ & 0.000 \\
\hline Visual impairment & $38(55.1 \%)$ & $52(28.7 \%)^{* *}$ & 0.000 \\
\hline Arthritis & $48(69.6 \%)$ & $62(34.3 \%)^{* *}$ & 0.000 \\
\hline Depression & $17(24.6 \%)$ & $18(9.9 \%)^{*}$ & 0.004 \\
\hline Hearing impairment & $20(29 \%)$ & $39(21.5 \%)$ & 0.244 \\
\hline Incontinence & $22(31.9 \%)$ & $17(9.4 \%)^{* *}$ & 0.000 \\
\hline Polypharmacy & $37(53.6 \%)$ & $55(30.4 \%)^{* *}$ & 0.001 \\
\hline Postural hypotension & $21(30.4 \%)$ & $2(1.1 \%)^{* *}$ & 0.000 \\
\hline Hand grip (kg) & $16.71 \pm 4.839$ & $18.03 \pm 4.185^{*}$ & 0.034 \\
\hline CST & $8.01 \pm 2.927$ & $10.69 \pm 2.486^{* *}$ & 0.000 \\
\hline TUG (seconds) & $13.10 \pm 4.390$ & $9.77 \pm 2.415^{* *}$ & 0.000 \\
\hline POMA & $22.81 \pm 2.927$ & $25.10 \pm 1.708^{* *}$ & 0.000 \\
\hline$\geq$ four risk factors & $53(76.8 \%)$ & $32(17.7 \%)^{* * *}$ & 0.000 \\
\hline
\end{tabular}

BMI=body mass index, CST=chair standing test, TUG=timed up and go,

POMA=performance-oriented mobility assessment

*Represents $p$ value $\leq 0.05$

**Represents $p$ value $\leq 0.001$

TABLE 2. TUG $v s$ falls

\begin{tabular}{|l|l|l|l|}
\hline TUG & Fallers $(\geq \mathbf{1}$ fall) $\mathbf{N = 6 9}$ & Non fallers $\mathbf{N}=\mathbf{1 8 1}$ & P value \\
\hline YES & $38(55.1 \%)$ & $23(12.7 \%)^{* * *}$ & 0.000 \\
\hline NO & $31(44.9 \%)$ & $158(87.3 \%)$ & \\
\hline
\end{tabular}

TUG=timed up and go

**Represents $p$ value $\leq 0.001$

TAB LE 3. POMA vs falls

\begin{tabular}{|l|l|l|l|}
\hline POMA & $\begin{array}{l}\text { Fallers }(\geq \mathbf{1} \text { fall) } \\
\mathbf{N}=\mathbf{6 9}\end{array}$ & $\begin{array}{l}\text { Non fallers } \\
\mathbf{N}=\mathbf{1 8 1}\end{array}$ & p value \\
\hline Low & $25(36.2)$ & $147(81.2 \%)^{* *}$ & 0.000 \\
\hline Medium & $34(49.3 \%)$ & $31(17.1 \%)$ & \\
\hline High & $10(14.5 \%)$ & $3(1.7 \%)$ & \\
\hline
\end{tabular}

TA B LE 4. Binary logistic regression of risk factors for fall

\begin{tabular}{|l|l|l|}
\hline Characteristics & Odds ratio $(\mathbf{9 5} \%$ CI) & p value \\
\hline Age $>80$ years & $3.8(1.8-8.1)^{* *}$ & 0.000 \\
\hline Female sex & $19.2(3.03-122.2)^{*}$ & 0.002 \\
\hline Postural hypotension & $59.8(3.3-1.07)^{*}$ & 0.006 \\
\hline Visual impairment & $8.9(1.4-55.4)^{*}$ & 0.019 \\
\hline$\geq$ four risk factors & $9.6(4.6-2.01)^{* *}$ & 0.000 \\
\hline
\end{tabular}

*Represents p value $\leq 0.05$

$* *$ Represents $p$ value $\leq 0.001$

(30.4\%) were significantly greater among fallers, who also had significant polypharmacy (53.6\%), more than two comorbidities (15.9\%), and more than three risk factors $(76.8 \%)$. Handgrip, CST score, and POMA score were found to be significantly low in fallers. The TUG score was significantly greater in fallers (Table 1 ).

The Fallers had significant TUG positivity (Table 2). According to POMA classification of risks, the medium and high-risk groups had significant fall (Table 3).

Binary logistic regression analysis showed that age over 80, female gender, and the presence of postural hypotension had an odds ratio (OR) of 3.8, 19.2 and 59.8, respectively. Visual impairment and the presence of $\geq$ four risk factors had an OR of 8.9 and 9.6, respectively. These five factors were significantly associated with an increased fall rate (Table 4).

\section{DISCUSSION}

-alls are a common challenge in the elderly that is associated with substantial morbidity and mortality. Falls often lead to functional impairment and to nursing home admissions. The prevalence of falls had a considerable variation among countries. In India, the prevalence of falls ranges from $14 \%$ to $53 \%$ due to underreporting, geographical region, criteria for fall, and methods to analyse falls. Fall-related injuries are also high in India (7-10).

In our study, 250 patients aged $\geq 60$ years attending the General Medicine outpatient department (OPD) were selected and examined. The prevalence of falls in our study was $27.6 \%(n=69)$. Subjects' mean age was $72.22 \pm 6.219$ years in the faller group and $70.36 \pm 4.668$ years in the non-faller one. A significant difference was no- 
ted between the two groups $(P=0.002)$. The prevalence of fall increased with age, as suggested by various studies (18-20). In our study, women $(75.4 \%)$ were significantly more prone to fall than men $(24.6 \%) \quad(P=0.001)$, which was supported by other similar studies $(21,22)$. In the present study, $15.9 \%$ of fall group population had more than three co-morbidities $(\mathrm{P}=0.029)$, including diabetes mellitus, hypertension, coronary artery disease, chronic kidney disease, chronic obstructive airway disease, bronchial asthma, and acid peptic disease. Fall risk was higher with an increasing number of comorbidities, similarly to the studies conducted by Paliwal $\mathrm{Y}$ et al and Vu T et al $(23,24)$.

In our study, $30.4 \%(n=21)$ of the faller group had fractures $(\mathrm{P}=0.000)$. Lower limb fracture $(24.6 \%)$ was the commonest followed by upper limb fracture (5.8\%). Hip fracture was the commonest in our study, similarly to the study performed by Shah Rashmi et al (25). In another study (26), the risk of osteoporotic fracture leading to fall was $9 \%$. Osteoarthritis is a common problem among the elderly. Knee arthritis is associated with poor balance and increases fall risk. Arthritis was the common risk factor for fall in our study $(69.6 \%$ compared to $34.35 \%$ in the non-faller group; $\mathrm{P}=0.000)$, similarly to findings reported by Krishnaswamy et al (27). In this study, osteoarthritis of the knee (56.5\%) was the most common one, followed by hip (8.7\%). Depression was seen in $24.6 \%$ of the fall group $(\mathrm{P}=0.004)$. Our findings correlate with those reported by Padubidri and Sirohi et al $(19,28)$. Hearing impairment was an insignificant risk factor for fall in our study in contrast to Sirhohi et al (28). Incontinence was significantly higher in the fall group (31.9\%; $\mathrm{P}=0.000)$, similarly to Dokuzlar O et al (29).

Polypharmacy is common in the elderly due to multiple co-morbidities. Thus, $53.6 \%$ of the fall group had polypharmacy compared to $30.4 \%$ in the non-faller group $(\mathrm{P}=0.001)$. Antihypertensives, oral hypoglycaemic agents, antiplatelets, pain killers, and psychotropic drugs were commonly used drugs in this study. In Anderson et al's study, fall-related medication was $41 \%$ (30). Tripathy et al stated that polypharmacy was significantly associated with falls (31). Hence, falls can be reduced by proper medication review. Visual impairment was seen in $55.1 \%$ of the faller group and was significantly associated with fall $(P=0.000)$ in this study, as suggested by Venkatesh $\mathrm{M}$ et al and Sirohi et al $(21,28)$. Postural hypotension was significantly higher in the faller group (30.4\%; $\mathrm{P}=0.000)$, which is comparable to the study by Gangavathi A et al. (32).

Thirty seconds chair standing test was significantly lower in the faller group $(8.01 \pm 2.927$; $\mathrm{P}=0.000)$. Chair standing test assesses the lower body muscle strength which is essential for functional performance in the elderly. Lesser counts in 30 seconds chair standing test were associated with a high risk of fall, as suggested by Narintip $R$ et al (16). Handgrip was significantly weaker in the faller group $(\mathrm{P}=0.034)$. Poor hand grip was significantly associated with fall in Yang et al's study (33).

Fall risk is high when the duration of TUG performance increases $\geq 12$ seconds. The mean duration to perform the TUG test was significantly higher in the faller group $(P=0.000)$; TUG was positive in $55.1 \%$ of the faller group in comparison with $12.7 \%$ of the non-faller group $(\mathrm{P}=0.000)$. The mean POMA score was significantly low in the faller group $(\mathrm{P}=0.000) ; 14.5 \%$ of subjects in the faller group had a high POMA score and $49.3 \%$ a moderate one, which is significantly higher than the non-faller group $(\mathrm{P}=0.000)$. Dokulzar et al found a $36.5 \%$ and $11 \%$ fall risk by TUG and POMA method, respectively (29). Fall risk increases with the number of risk factors. In our study, $76.8 \%$ of subjects had more than three risk factors for fall $(P=0.000)$.

On binary logistic regression, we found a significant fall rate with the following risk factors: age $>80$ years, female gender, visual impairment, postural hypotension, and presence of more than three risk factors. Normal gait requires a good coordination with fine neural networks, exquisite musculoskeletal structures along cognition. Aging-related decline in these functions along with decrease in a gait velocity, wider base, and decreased muscle strength increases the risk of fall in the elderly populations. This is further exaggerated by multiple co-morbid conditions and drugs in the elderly. Patients aged over 80 $(15.9 \%)$ had a significant fall rate in our study $(\mathrm{OR}=3.8 ; \mathrm{P}=0.000)$. In a study by Chacko et al, age $>80$ was a significant risk factor $(\mathrm{OR}=3.28)$ (18). Alshmmari et al have also stated that patients' age was a significant risk factor for fall (22). Female gender is also a well-known risk factor for fall. In this study, females had a signifi- 
cantly higher fall rate than their counterparts (75.4\%; $\mathrm{OR}=19.2 ; \mathrm{P}=0.002)$, which may be due to the fact that women's increased life expectancy versus men leads to a higher longevity in women. Our finding was very well supported by other studies $(21,22)$.

Visual impairment is seen in $55.1 \%$ of the faller group in comparison to the $28.7 \%$ of the non-faller group $(\mathrm{OR}=8.9, \mathrm{P}=0.019)$. Dhargave et al found a significant relation between poor vision and fall risk $(\mathrm{OR}=1.81 ; \mathrm{P}<0.005)$ (34). The study of Sirohi et al on an elderly population including 467 persons found that fall risk was increased with visual impairment $(\mathrm{OR}=1.6$; $\mathrm{P}=0.049$ ) (28). Aging-induced diminution of baroreceptor response and vasoconstriction, slow cardiac filling, and impaired renal salt and water conservation increase the risk of postural hypotension in the elderly. Postural hypotension leads to transient cerebral ischemia, which may worsen a chronic decrease in cerebral blood flow and subsequently lead to falls. Postural hypotension was significantly associated with fall in our study by logistic regression $(O R=59.8$; $\mathrm{P}=0.000)$. This finding was supported by other studies too $(32,35)$.
Fall risk in the elderly increases exponentially with the number of risk factors such as musculoskeletal, neurological, functional dependency, psychosocial, and drug use. In our study, 76.4\% of subjects in the faller group had more than three risk factors compared to $17.7 \%$ in the non-faller group. 'Number of risk factors' was the foremost risk for fall in our study. Lawler DA et al also stated that fall risk increases in proportion to the number of risk factors (20).

\section{Study limitations}

Our study was conducted in the OPD and included only active elderly patients.

\section{CONCLUSIONS}

\begin{abstract}
-alls among older populations were common - especially in persons aged over 80 and females. Fall rate increased linearly with the number of risk factors and simple clinical assessments, including TUG, POMA and 30 seconds CST, are useful to identify high-risk patients.
\end{abstract}

Conflicts of interest: none declared. Financial support: none declared.

\section{R RFERENCES}

1. Census of India for 1961-2001, NSSO Survey 2004-05 and 2007-08, SRS (Sample Registration System) Statistical Report 2011.

2. Population Reference Bureau. World population highlights: key findings from PRB's 2010 world population data sheet. Available at: http://www.prb.org/pdf10/65.2highlights. pdf.

3. SRS Bulletin (Sample Registration System) October, 2012, Registrar General of India, New Delhi.

4. Graafmans WC, Ooms ME, Hofstee HMA, et al. Falls in the Elderly: A Prospective Study of Risk Factors and Risk Profiles. American Journal of Epidemiology 1996;143:1129-1136.

5. Hsu HC, Jhan LJ. Risk Factors of Falling among the Elderly in Taiwan: A Longitudinal Study.

Taiwan Geriatrics $\mathcal{E}$ Gerontolog 2008;3:141-154.

6. Jagnoor J, Wilson S, Keaya L, et al. Childhood and adult mortality from unintentional falls in India. Bulletin of the World Health Organization 2011;89:733-740.
7. Dsouza SA, Rajashekar B, Dsouza HS, Kumar KB. Falls in Indian older adults: A barrier to active aging. Asian J Gerontol Geriatr 2014;9:33-40

8. Johnson SJ. Frequency and nature of falls among older women in India. Asia Pac J Public Health 2006;18:56-61.

9. Joshi K, Kumar R, Avasthi A. Morbidity profile and its relationship with disability and psychological distress among elderly people in Northern India. Int J Epidemiol 2003;32:978-987.

10. Krishnaswamy B, Gnanasambandam U. Falls in older people. National/regional review, India. Available at: http://www.who.int/ aging/projects/ SEARO.pdf.

11. Prevention AGSPF: Guideline for the prevention of falls in older persons. American Geriatrics Society, British GeriatricSociety, and American Academy of Orthopaedic SurgeonPanel on Falls Prevention. I Am Geriatr Soc 2001;49:664-672.

12. Brown AP. Reducing falls in elderly people: A review of exercise interventions. Physiother Theory Pract1999;15:59-68.

13. Taro Kojima. The Need for Actions
Against Polypharmacy in Older People With Frailty. Annals of Geriatric Medicine and Research 2018;22:111-116

14. WHO Expert Consultation Appropriate body mass index for Asia populations and its implications for policy and intervention strategies. Lancet 2004;363:157-163.

15. Mathias $S$, Nayak US, Isaacs $B$. Balance in elderly patients:the "Get-up and $\mathrm{Go}^{\prime \prime}$ test.

Arch Phys Med Rehabil 1986;67:387-389.

16. Narintip Roongbenjawan, Akkradate Siriphorn. Accuracy of modified 30-s chair-stand test for predicting falls in older adults. Annals of Physical and Rehabilitation Medicine 2020;63:309-315.

17. Amaral CA, Amaral TLM, Monteiro GTR, et al. Hand grip strength: Reference values for adults and elderly people of Rio Branco, Acre, Brazil. PLoS One 201931;14:e0211452.

18. Chacko TV, Thangaraj $P$, Muhammad GM. Epidemiology of fall and its risk factors among elders in a rural area of Coimbatore, India. Int J Community Med Public Health 
2017;4:3864-3869.

19. Padubidri A, Al Snih S,

Samper-Ternent $R$, et al. Falls and cognitive decline in Mexican Americans 75 years and older. Clin Interv Aging 2014;9:719-26. D'Souza, S. A.

Circumstances and Consequences of Falls in Indian Older Adults. The Indian Journal of Occupational Therapy 2008;40:7-9.

20. Lawlor DA, Patel R, Ebrahim S. Association between falls in elderly women and chronic diseases and drug use: cross sectional study. BMJ 2003;327:712-717.

21. Venkatesha M, Latha K, Mokhasi VR, et al. Prevalence and Risk for Fall among Elderly in Urban Area of Southern Karnataka. Ntl J Community Med 2016;7:768-771.

22. Alshammari SA, Alhassan AM, Aldawsari MA, et al. Falls among elderly and its relation with their health problems and surrounding environmental factors in Riyadh. J Family Community Med 2018;25:29-34

23. Paliwal Y, Slattum PW, Ratliff SM. Chronic Health Conditions as a Risk Factor for Falls among the CommunityDwelling US Older Adults: A Zero-Inflated Regression Modeling Approach.
Biomed Res Int 2017;2017:5146378.

24. Vu T, Finch CF, Day L. Patterns of comorbidity in community-dwelling older people hospitalised for fall-related injury: a cluster analysis. BMC Geriatr 2011;11:45.

25. Shah Rashmi S, Savardekar LS. Post menopausal osteoporosis in India: growing public health concern. National Institute for Research in Reproductive Health, India, Mumbai, 2005, pp. 12-16.

26. Naumov AV, Khovasova NO, Moroz VI, Tkacheva ON. Falls and pathology of the musculoskeletal system in the older age groups.

Zh Nevrol Psikhiatr Im S S Korsakova 2020;120:7-14.

27. Krishnaswamy B, Shanthi, G. Risk factors for falls in the elderly. Journal of Indian Academy of Geriatrics 2005;1:57-60.

28. Sirohi A, Kaur R, Goswami AK, et al. Astudy of falls among elderly persons in a rural area of Haryana. Indian J Public Health 2017;61:99-104.

29. Dokuzlar O, Koc Okudur S, Smith L, et al. Assessment of factors that increase risk of falling in older women by four different clinical methods. Aging Clin Exp Res 2020;32:483-490.

30. Andersen CU, Lassen PO, Usman HQ, et al. Prevalence of medication-related falls in 200 consecutive elderly patients with hip fractures: a cross-sectional study. BMC Geriatr 2020;20:121.

31. Tripathy NK, Jagnoor J, Patro BK, et al. Epidemiology of falls among older adults: A cross sectional study from Chandigarh, India. Injury 2015;46:1801-1805.

32. Gangavati A, Hajjar I, Quach L, et al. Hypertension, orthostatic hypotension, and the risk of falls in a communitydwelling elderly population: the maintenance of balance, independent living, intellect, and zest in the elderly of Boston study.

J Am Geriatr Soc 2011;59:383-389. Erratum in: J Am Geriatr Soc 2011;59:960.

33. Yang NP, Hsu NW, Lin CH, et al. Relationship between muscle strength and fall episodes among the elderly: the Yilan study, Taiwan. BMC Geriatr 2018;18:90.

34. Dhargave $P$, Sendhilkumar R. Prevalence of risk factors for falls among elderly people living in longterm care homes.

J Clin Gerontol Geriatr 2016;7:99-103.

35. Mol A, Bui Hoang PTS, Sharmin S, et al. Orthostatic Hypotension and Falls in Older Adults: A Systematic Review and Meta-analysis.

J Am Med Dir Assoc 2019;20:589-597.e5. 